Journal of

Synchrotron

Radiation

ISSN 0909-0495

Received 15 October 2004

Accepted 1 February 2005

(C) 2005 International Union of Crystallography

Printed in Great Britain - all rights reserved

\section{Microfocus X-ray scattering investigations of eggshell nanotexture}

\author{
Donna Lammie, ${ }^{\mathrm{a}, \mathrm{b} *}$ Maureen M. Bain ${ }^{\mathrm{a}}$ and Tim J. Wess ${ }^{\mathrm{b}}$ \\ ${ }^{\mathbf{a}}$ Division of Cell Sciences, Institute of Comparative Medicine, Faculty of Veterinary Medicine, \\ University of Glasgow, Bearsden Road, Glasgow G61 1QH, UK, and ${ }^{\mathbf{b}}$ Biophysics Group, School of \\ Optometry and Vision Sciences, Cardiff University, Redwood Building, King Edward VII Avenue, \\ Cathays Park, Cardiff CF10 3NB, UK. E-mail: lammied@cf.ac.uk
}

\begin{abstract}
The avian eggshell is a highly ordered calcitic bioceramic composite, with both inorganic and organic constituents. The interactions between the inorganic and organic components within the structure are poorly understood but are likely to occur at the nanometre level. Thus structural variation at this level may impinge on the overall structural integrity and mechanical performance of the eggshell, and therefore analysis at this level is fundamental in fully understanding this ordered structure. In this study, structural changes in the mineral crystallites were investigated by microfocus small-angle X-ray scattering ( $\mu$ SAXS) using synchrotron radiation. Small-angle X-ray scattering (SAXS) can be used to investigate structures on the nanometre scale such as size, shape, arrangement and internal porosity. A microfocused X-ray beam, $1.5 \mu \mathrm{m}$ vertically by $7 \mu \mathrm{m}$, was used to produce vertical linear scans of the eggshell section. SAXS patterns were taken from the eggshell membrane (inner surface of the eggshell) to the cuticle (outer surface of the eggshell). This allowed textural variations within the eggshell to be mapped. The scattering intensity profile was then used to derive the dimension of scattering objects that define the nanotexture. The nanotexture observed may result from the presence of the organic matrix, which is embedded as intracrystalline particles producing voids within the calcified framework of large $(>1 \mu \mathrm{m})$ calcite crystals. Porod analysis revealed the average size of a scattering interface to be approximately $4.5 \mathrm{~nm}$ with small changes that had a depth-dependent variation. These were largest at the mammillary layer/ membrane boundary. The palisade layer displayed a small upward trend in size of scattering object. Parallel scans showed that the textural variations observed within the palisade layer are significant and indicate local subtextures. In addition, many of the patterns exhibit diffuse scattering streaks that could result from reflectivity from the larger crystallite interfaces. Changes in the orientation of diffuse streaks were observed within the different layers, the membranes, mammillary layer, palisade layer, vertical crystal layer and cuticle, indicating certain preferred orientations of the crystallites within the layers. The nanotextural variations that are apparent could have implications at the macroscopic level of the resulting eggshell.
\end{abstract}

Keywords: microfocus small-angle X-ray scattering; eggshell; structure; nanotexture.

\section{Introduction}

The field of biomineralization has been widely studied in many biological systems; for example, biological calcification can be seen in the formation of bones, teeth, corals, mollusc shells and eggshells. The calcification process of the avian eggshell is one of the fastest known and is complete in approximately $24 \mathrm{~h}$.

The eggshell is a highly ordered structure (Solomon, 1991; Parsons, 1982) and comprises five structurally different layers. These include, from the inside outwards, the shell membranes (inner and outer), mammillary layer, palisade layer, vertical crystal layer and cuticle (Fig. 1). From the inner membranes, the first mineralized layer is the mammillary layer, thought to contain randomly orientated crystals (Cain \& Heyn, 1964). The calcite crystal sizes are large, $>1 \mu \mathrm{m}$, as evidenced from a number of studies including analysis using the Rietveld method (T. Wess, unpublished data) and also powder line broadening. These studies all indicate that the sizes are beyond the small-angle X-ray scattering (SAXS) range determination, which is ideally between $\sim 1$ and $50 \mathrm{~nm}$. The 


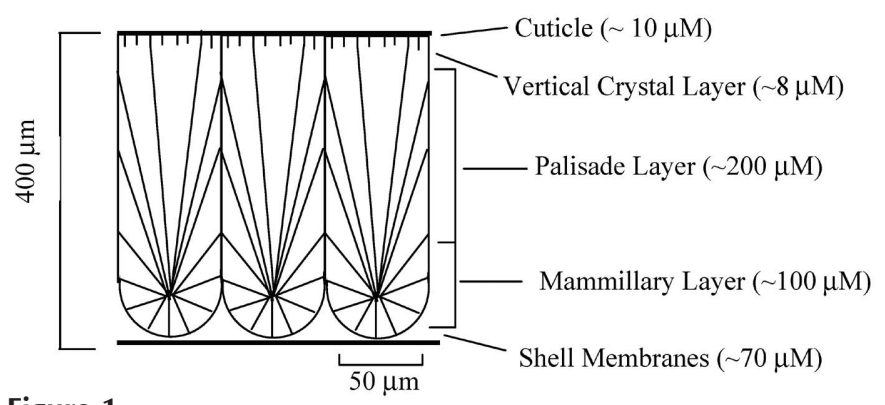

Figure 1

Schematic diagram of the five structurally different layers within the eggshell with the inside at the bottom and the outside at the top. The lines indicate a preferred orientation of calcitic crystal texture.

palisade layer makes up the majority of the calcified portion of the shell; here the calcite crystals grow with a long aspect perpendicular to the surface (Parsons, 1982). The palisade layer is continued as the vertical crystal layer, which is a thin layer immediately underneath the cuticle.

The eggshell is composed of approximately $95 \%$ calcium carbonate in the form of calcite and approximately $3.5 \%$ proteins, proteoglycans and glycoproteins (Nys et al., 1999; Arias et al., 1993). The exact role played by the organic components in eggshell formation is not well understood; however, they are thought to influence nucleation, control crystal growth and shape, and play a role in determining the mechanical properties of the resulting composite, as has been suggested in other biological systems (Addadi \& Weiner, 1992). The eggshell forms in the shell gland of the oviduct in an acellular milieu which is supersaturated with calcium and bicarbonate ions and which also contains a range of proteins that are essential for eggshell formation (Nys et al., 1999; Arias et al., 1993). There are three different phases of eggshell production: initial, growth and terminal, and each of these stages is characterized by differing amounts and types of protein within the uterine fluid. Examples of specific proteins present at the different phases include ovalbumin, ovotransferrin and lysozyme at the initial phase. Ovocleidin-17 and ovocleidin-116 are examples of two proteins that are present at the growth phase and the protein ovocalyxin- 32 at the terminal phase. These proteins can broadly be divided into insoluble and soluble proteins; the insoluble proteins (intermineral) are thought to act as a structural framework, and the soluble proteins (intramineral) during calcification become embedded within the crystal.

Structural hierarchies are common in biology and conformation at the nanoscale frequently influences macroscopic properties. SAXS is a technique capable of analysing size, shape, arrangement and internal porosity of nanostructures. Scattering data provide accurate values for many structural parameters because the data enable analysis over large volumes of the sample. The intensity distribution of the scattered X-rays provides information on average particle thickness and crystal shape. Microfocus-SAXS ( $\mu$ SAXS) is an adaptation of SAXS that allows textural variation in micrometre-sized volumes of the sample to be investigated. Owing to the intense micro X-ray beam, this has permitted nano- textural mapping of many samples that have variations in texture. Such samples include bone (Wess, Alberts et al., 2001), calcified avian tendons (Gupta et al., 2003), parchment (Kennedy et al., 2004) and starch granules (Waigh et al., 1997).

In this study we used $\mu \mathrm{SAXS}$ to investigate variations in nanostructures within the eggshell of Gallus gallus. The calcitic crystals that comprise the eggshell are considered to influence its structure at the microscopic level; however, here we probe the interactions between protein and mineral that are expected to occur at a nanoscopic level. Since a number of different proteins are expressed during development, we wished to examine whether their relationship with the mineral phase could be detected by SAXS. This level of detail could further our knowledge of the relationship between the organic material and the microstructure.

\section{Materials and methods}

\subsection{Sample preparation}

In this study, high-quality eggshells (determined by their breaking strength) from commercial breeding lines of Gallus gallus were investigated. Equatorial sections of eggshells were impregnated in a polyester resin system, using Crystic resin with butan-1-one peroxide and cobalt octoate catalyst. The resin was mixed with acetone and degassed prior to vacuum impregnation. After curing, $300 \mu \mathrm{m}$-thick sections were cut from each sample using a Beuhler isomet low-speed saw with diamond wavering blade; rape seed oil was used as the cutting fluid.

\subsection{Microfocus $X$-ray scattering}

Microfocus analysis was carried out on beamline ID18F at the European Synchrotron Radiation Facility, Grenoble, France, where a compound refractive lens (Lengeler et al., 1999) was used to generate a $1.5 \mu \mathrm{m} \times 7 \mu \mathrm{m}$ (FWHM) microbeam. A detailed description of the instrumentation setup is described by Wess, Alberts et al. (2001). The sample-todetector distance was $20 \mathrm{~cm}$ and the wavelength was $0.086 \mathrm{~nm}$. To absorb the direct beam a tungsten backstop of diameter $500 \mu \mathrm{m}$ was used. The detector pixel size was $150 \mu \mathrm{m}$ both horizontally and vertically. This defined the $\mathbf{q}$ scattering limits to be $0.187-2.00 \mathrm{~nm}^{-1}$, where $\mathbf{q}$ is the scattering vector equal to $4 \pi \sin \theta / \lambda$. The eggshell samples were mounted on a goniometer controlled remotely on a motorized stage; the samples were aligned perpendicular to the X-ray beam. The microfocus apparatus was removed and, using the defocused beam and a high-resolution camera, an X-ray transmission image was recorded of each of the eggshells to observe the microscopic features (Fig. 2).

Using the microfocused beam, the eggshell sections were scanned vertically through three sections of three separate eggs, from the inner eggshell surface to the outer surface. In each case the total scan length was $500 \mu \mathrm{m}$, and 31 measurements were made at $16 \mu \mathrm{m}$ intervals and repeated a further two times at $48 \mu \mathrm{m}$ intervals (Fig. 3). The overall scan 


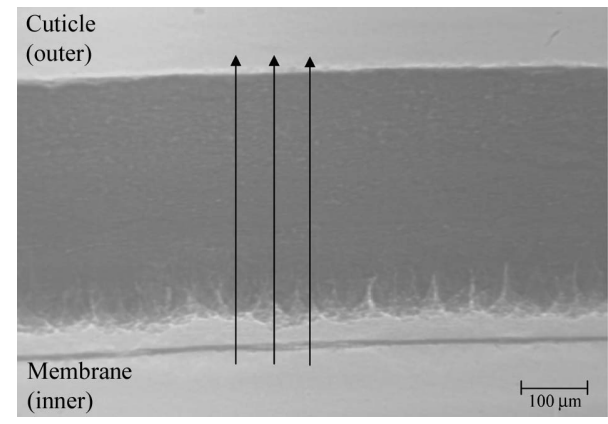

Figure 2

A typical X-ray transmission image of an eggshell, obtained using the defocused beam. The arrows shows the areas where X-ray scattering patterns were recorded: three vertical scans were made $48 \mu \mathrm{m}$ apart. The vertical scan length was $500 \mu \mathrm{m}$. Samples were bathed in the direct beam. Differential absorbance allows microscopic features to be observed.

dimensions ensured that the whole eggshell section was sampled from the inner surface to the outer surface.

\subsection{Data processing}

The two-dimensional SAXS patterns which were collected at ID18F were spherically averaged to produce one-dimensional linear profiles, $I(\mathbf{q})$. The linear profiles were corrected for transmission, and background was subtracted as described by Wess, Drakopoulos et al. (2001). Log-log plots were produced (Fig. 4) which show power-law decays in intensity, where the knee region gave an indicative size measurement of the scattering element. From the linear profiles, the invariant (Fig. 5) and Porod constant were determined, where the invariant is the total scatter extrapolated to $\mathbf{q}=0$ and $\mathbf{q}=\infty$. This was used to determine the smallest dimension of the scattering objects after Fratzl et al. (1996), which allowed the ratio of the surface area to the volume to be determined, giving information on the crystallite-protein relationship. The data exhibited an isotropic scatter; superimposed on this in some cases were diffuse streaks of scatter attributed to reflectivity of the large calcitic crystallites. The orientation of the crystallites was deduced by examining the angular distribution to highlight any changes between the different layers and from one vertical linear scan to the other.

\section{Results and discussion}

The X-ray transmission image of the eggshell using the defocused beam shows the textural variation within the calcified eggshell (Fig. 2), and is most noticeable at the inner face of the shell where the mammillary region contains a complex microtexture. In transmission, the palisade layer shows a more homogeneous texture although microvoids of porosity can be seen to occur with a greater frequency towards the cuticle. Nanostructural detail cannot, however, be produced using such a technique, but this image serves to indicate the representative scan regions used in this work. The line from the inner membrane to the outer cuticle highlights the direction of the linear X-ray scattering scan. This indicates the area that was scanned for reference purposes. Fig. 3 shows a composite of the linear SAXS patterns that were observed, proceeding from the membrane (inner surface) to the cuticle (outer surface). The first three SAXS patterns and the last two SAXS patterns correspond to the embedding resin. This ensures that the full length of the eggshell was scanned. The change in the total intensity of the scattering profiles is shown in Fig. 5 indicating that the strength of scattering increases within the palisade layer.

A dimension of the scattering object was derived from this study; the variation of this dimension was examined throughout the length of the scan, corresponding to the different layers of the eggshell. A value was derived for each of the individual scattering patterns; these values are plotted on Fig. 6. The average dimension for the scattering object was found to be approximately $4.5 \mathrm{~nm}$. Points $1-3$ on the scan and points 30 and 31 are values from the resin, within which the sample is embedded; as such, the dimensions are not valid for this study. Similar trends can be seen when comparing the values obtained from the three scans. There is a high increase in size value at point 4 in two of the scans which corresponds to the SAXS pattern that also shows preferred orientation, an anisotropic scattering distribution. This we believe corresponds to the detached inner membrane that also contains mineral components attached to the fibres. Dimensional measurements show a decrease between the membrane and the mineral portion of the eggshell in these two scans; this could indicate that the inner membrane has separated from the rest of the eggshell during the embedding process, which would account for the gap in intense scattering. The third scan, shown as triangles in Fig. 6, does not show an increase in value at point 4 , indicating that the detached inner membrane may have been missed in the scan. The mammillary layer contains approximately $66 \%$ of the non-membrane intercrystalline and intracrystalline organic material (Carter, 1969). This region also produced the strongest SAXS signal indicating that proteins may act as X-ray lucent voids especially in this region. The greater portion of the bulk measurements from the palisade layer show small fluctuations in particle size but in general the size is between 4 and $5 \mathrm{~nm}$. Fig. 6 shows superimposed pore diameters values from three adjacent scans indicating that the fluctuations observed are reproducible and significant. We believe that these changes in pore size may correspond to local alterations in the complement of proteins expressed and deposited at different times during eggshell development in the oviduct. This indicates for the first time that there is a significant nanotexture with eggshell which is not accounted for by previous transmission electron microscopy (TEM) and high-angle diffraction studies.

The ability to indicate that the nanotexture exhibits some variation corresponding to different structural layers is also an important addition to our knowledge of the structure of eggshell over a range of length scales from the atomic to macroscopic. In an analysis of texture, Perrott et al. (1981) found using TEM and diffraction that the calcite columns within the palisade layer consisted of crystallites of diameter 20-30 $\mu \mathrm{m}$. Also, Cain \& Heyn (1964) found the mineral particle sizes to be $200 \mu \mathrm{m}$ by using the number of diffraction 


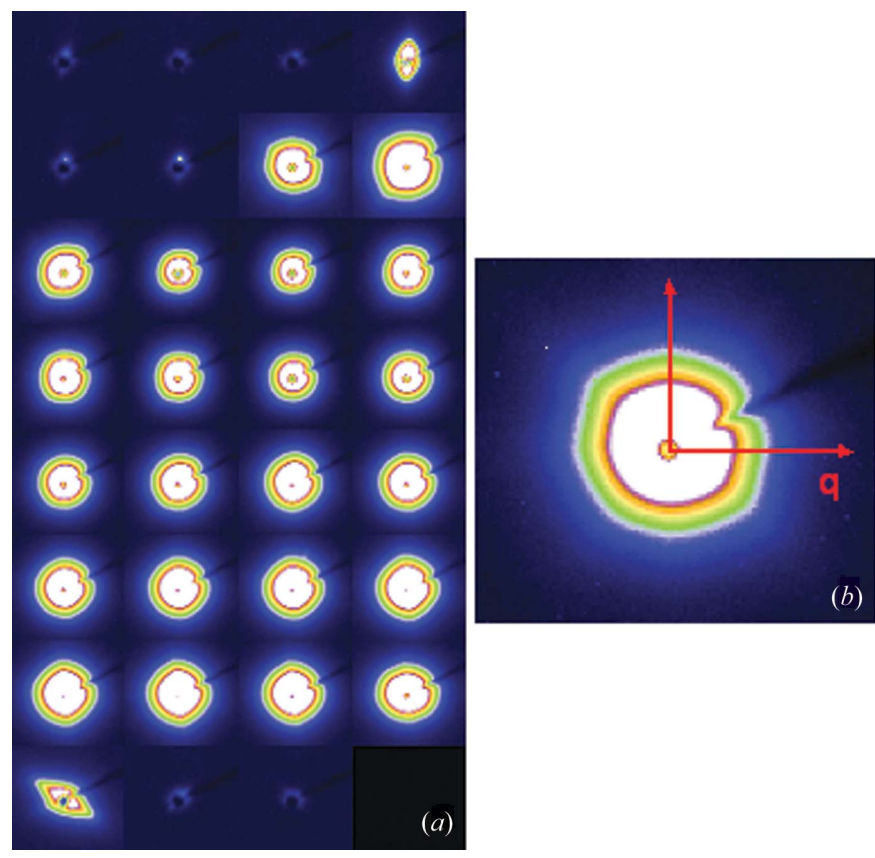

Figure 3

(a) Composite of the X-ray patterns that were obtained from the vertical scan through the eggshell section. The patterns are arranged in rows from the top left to the bottom right, where the first pattern corresponds to the resin at the inner surface of the eggshell and proceeds to the outer surface. (b) An individual X-ray scattering pattern from the composite, which shows a strong isotropic scatter and the presence of diffuse streaks of scatter.

spots in the rings to determine the crystallite size. The overall sizes of the calcite crystals are known to be too large for accurate detection using $\mu$ SAXS because the size of the beam is smaller than the size of the crystal. The measurements that are presented here therefore constitute detail on the nanoscale that could represent the connection between detailed biochemical studies that indicate the presence of intercrystalline and intracrystalline proteins. Such proteins typically have a relative molecular mass in the range $30-50 \mathrm{KDa}$ which would be commensurate with a spheroid diameter of $3-5 \mathrm{~nm}$. Intracrystalline proteins are found at all stages of growth (initial, growth and terminal), with the majority at the later stages (Gautron et al., 1996); therefore these results are likely to be from the intracrystalline proteins embedded within the calcified framework. Using SAXS, however, it cannot be unequivocally confirmed that these voids account for the scattering signal; nanocrystallites of calcite by the inversion principal could also produce a similar effect.

In addition to the isotropic scattering, the diffuse streaks thought to be caused by reflectivity of the crystallite boundaries were examined to investigate changes in calcitic crystal orientation. These effects are most prominent at the start and end of the scans where the initial part of the membrane/ mammillary interface and the vertical crystal layer are sampled. The results presented here show that there are variations in crystallite orientation within the structurally different layers that comprise the eggshell, the membranes, mammillary layer, palisade layer, vertical crystal layer and

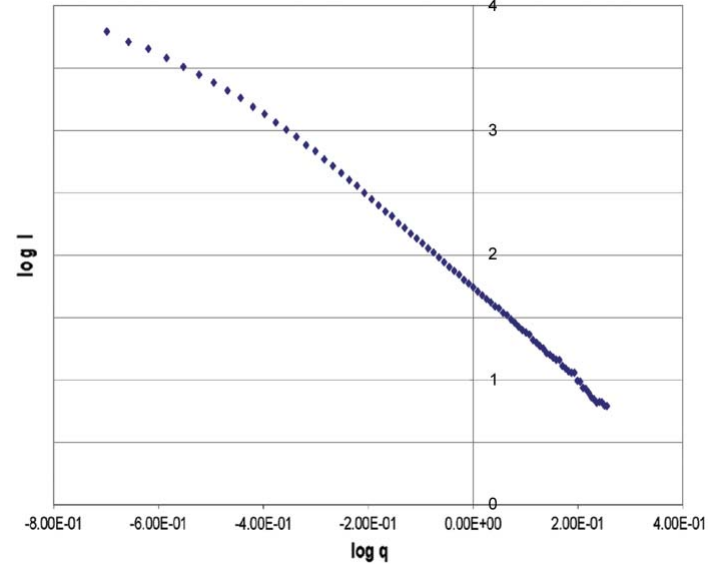

Figure 4

A $\log -\log$ plot presenting a linear profile from one of the scattering patterns within the vertical linear scan. This shows the power-law decaying in intensity, where the knee region gives an indicative size measurement of the scattering element.

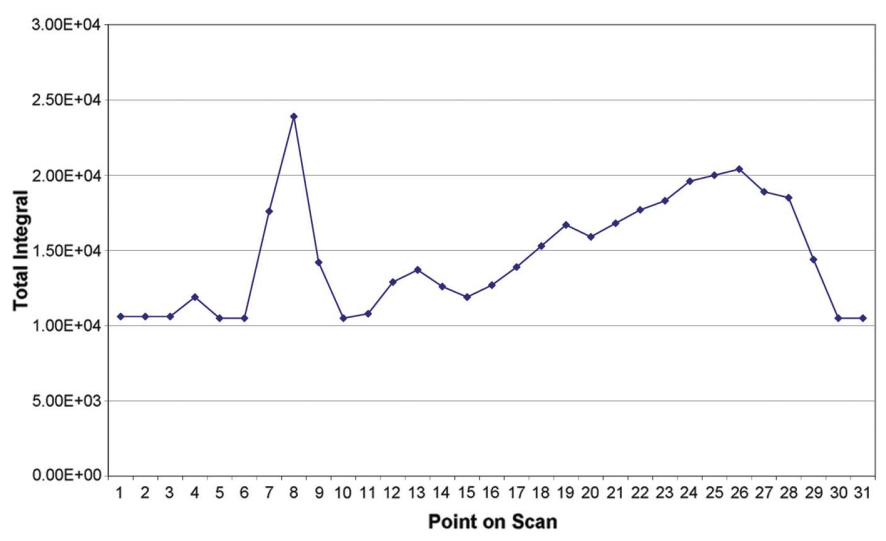

Figure 5

Total integral of scattering intensity at each of the points on the vertical scan. The largest value at point 8 corresponds to the centre of the mammillary layer. An increase in the total integral is seen as the scan progresses towards the outer surface of the shell. The sample thickness was $300 \mu \mathrm{m}$ throughout.

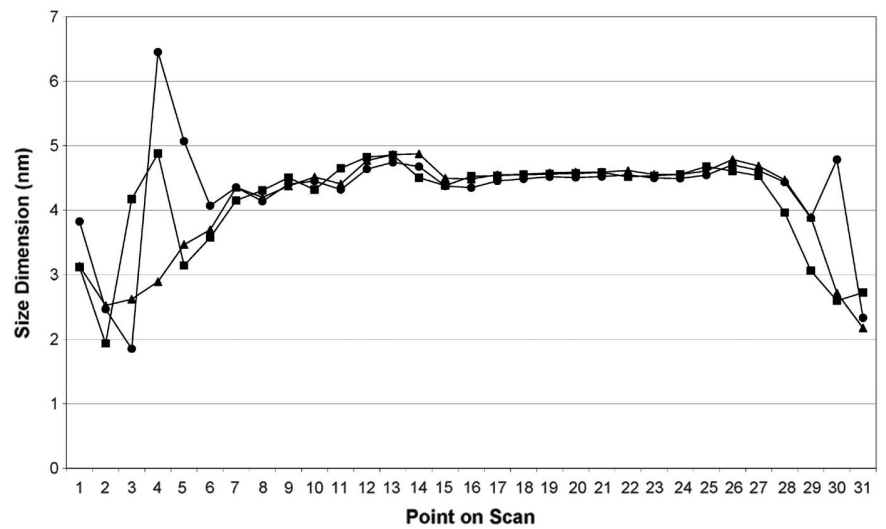

Figure 6

Measurements of the smallest dimension of the scattering objects from three scans on one eggshell; similar trends can be seen. The scans start at the inner membrane and finish at the outer surface cuticle. Resin measurements are also included at the beginning and at the end of the scans. 
cuticle. By examining the patterns of the scan it can be seen that, in the initial pattern from the eggshell, pattern 4, the detached membrane/mammillary layer interface exhibits a clear preferential orientation of scattering which is perpendicular to the tangent of the eggshell surface (Fig. 7a). This indicates that there are small crystallites within the membrane fibres that are aligned parallel to the shell surface, or that the membrane fibres themselves are acting as low-density components in a composite where the fibrous feltwork is parallel to the shell surface.

Towards the end of the vertical scan before the scan point re-entered the resin, the scattering pattern shows a preferred orientation indicated by the shape of the scattering distribution. This is probably not due to intracrystalline globular protein since spherical objects cannot present a preferred orientation. The scattering distribution has an approximately $36^{\circ}$ orientation to the shell tangent (Fig. $7 b$ ), therefore the long axis of scattering objects are orientated $90^{\circ}$ from the direction of the scattering distribution. This has been found in all the shells studied and is a feature that cannot easily be explained from conventional morphological studies. As the vertical crystal layer is approximately $8 \mu \mathrm{m}$ in length (Perrott
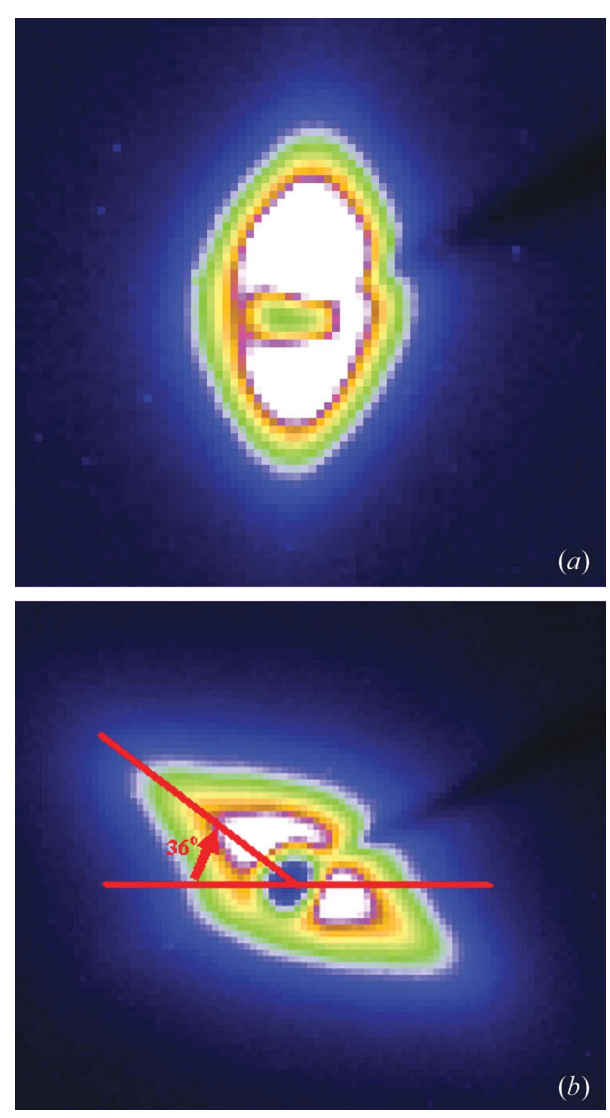

\section{Figure 7}

(a) SAXS pattern from the membranes/mammillary layer. The scatter distribution is perpendicular to the tangent of the eggshell surface. $(b)$ SAXS pattern at point 29 on the vertical scan from the vertical crystal layer/cuticle part of the eggshell. The scattering distribution is $36^{\circ}$ to the tangent of the eggshell surface. Comparison of both patterns indicates that at the inner and outer parts of the shell there is evidence of preferred orientation. et al.,1981), there is a chance that this area of the eggshell was not scanned owing to the size of the scanning steps used being $16 \mu \mathrm{m}$. However, as the cuticle is approximately $10 \mu \mathrm{m}$, at least one SAXS pattern was captured from these adjoining parts of the eggshell. Perrott et al. (1981) also stated that the only layer that showed any sort of preferred orientation was the surface crystal layer. This also coincides with the results here regarding the outer part of the shell showing a scattering distribution that indicates an orientation at approximately $36^{\circ}$.

\section{Future work}

Microfocus SAXS has proved to be an excellent tool for providing information about the structure and textural differences on the nanometre length scale of the eggshell structure. The strength properties of eggshell are renowned and are a paradigm of structure-function relationships; however, the molecular organization and interplay of organic and inorganic matrix are poorly understood. Until now, relatively little was known about nanoscale textures of this abundant mineralized tissue. The point-to-point resolution used in this pilot study shows that there is textural variation within the eggshell; however, the sampling of layers such as the vertical crystal layer and the interface with the cuticle could be improved upon by using nanobeam technology, where the structural changes that are better matched to the size of the morphological variation in the eggshell may be mapped in more detail. The relationship between the intercrystalline and intracrystalline proteins may be addressed in the future by attempting to contrast match the calcite crystal interfaces and voids between calcite crystals with a solution of similar electron density. If the intracrystalline proteins that are believed to be embedded in the crystals are not accessible to the contrast matching solution, then it can be confirmed that the scattering signal results from protein voids within the crystal matrix.

Thanks to Professor Sally Solomon for useful discussions and advice, and to Dr Michael Drakopoulos, formerly at the ESRF, for technical support. This work was carried out at the ESRF as part of a long-term beam-time proposal awarded to TJW. DL is supported by BBSRC.

\section{References}

Addadi, L. \& Weiner, S. (1992). Angew. Chem. Int. Ed. Engl. 31, 153169.

Arias, J. L., Fink, D. J., Xiao, S. Q., Heuer, A. H. \& Caplan, A. I. (1993). Int. Rev. Cytol. 145, 217-250.

Cain, C. J. \& Heyn, A. N. J. (1964). Biophys. J. 4, 23-39.

Carter, T. C. (1969). Br. Poul. Sci. 10, 165-174.

Fratzl, P., Schreiber, S. \& Klaushofer, K. (1996). Connect. Tissue Res. 34, 247-254.

Gautron, J., Bain, M., Solomon, S. \& Nys, Y. (1996). Br. Poult. Sci. 37, 853-866. 
Gupta, H. S., Roschger, P., Zizak, I., Fratzl-Zelman, N., Nader, A., Klaushofer, K. \& Fratzl, P. (2003). Calcif. Tissue Int. 72, 567576.

Kennedy, C. J., Hiller, J. C., Lammie, D., Drakopoulos, M., Vest, M., Cooper, M., Adderley, W. P. \& Wess, T. J. (2004). Nano Lett. 4, 1373-1380.

Lengeler, B., Schroer, C., Tummler, J., Benner, B., Richwin, M., Snigirev, A., Snigireva, I. \& Drakopoulos, M. (1999). J. Synchrotron Rad. 6, 1153-1167.

Nys, Y., Hincke, M. T., Arias, J. L., Garcia-Ruiz, J. M. \& Solomon, S. E. (1999). Poult. Avian Biol. Rev. 10, 143-166.
Parsons, A. H. (1982). Poult. Sci. 61, 2013-2021.

Perrott, H. R., Scott, V. D. \& Board, R. G. (1981). Calcif. Tissue Int. 33, 119-124.

Solomon, S. E. (1991). Egg and Eggshell Quality. London: Wolfe.

Waigh, T. A., Hopkinson, I., Donald, A. M., Butler, M., Heidelbach, F. \& Riekel, C. (1997). Macromolecules, 30, 3813-3820.

Wess, T. J., Alberts, I., Hiller, J., Drakopoulos, M., Chamberlain, A. T. \& Collins, M. (2001). Calcif. Tissue Int. 70, 103-110.

Wess, T. J., Drakopoulos, M., Snigirev, A., Wouters, J., Paris, O., Fratzl, P., Collins, M., Hiller, J. \& Nielsen, K. (2001). Archaeometry, 43, 117-129. 